P179 (continued)

distribution and redemption. Since the program's launch in May through October 2017, 77 individuals enrolled in the program from three participating pharmacies. On average, participants reported consuming 2.5 servings of healthy fruits and vegetables per day at baseline. The majority of participants (57\%) worried about having enough food to eat in the past 30 days.

Conclusions and Implications: Given the low average consumption of fruits and vegetables and inconsistent access to food, we anticipate this prescription program will have the intended benefit.

Funding: USDA.

\section{P180 Process Evaluation of a SNAP-Ed Healthy Corner Store Initiative in New York State}

Alisha Gaines,PhD, CHES, againes@cornell.edu, Cornell University, Division of Nutritional Sciences, 342A MVR Hall, Reservoir Avenue, Ithaca, NY 14853; Zoe Wakoff, MPH; Tisa F. Hill, MPH; Jamie S. Dollahite, PhD

Background (Background, Rationale, Prior Research, and/or Theory): To address poor access to healthful food, a SNAP-Ed healthy corner store policy, systems, and environment (PSE) initiative in New York State was designed to increase availability of fruits, vegetables, and low-fat dairy in local corner stores using phased changes, beginning with education and marketing strategies and ending with sustained increases in healthful offerings. Process evaluation was an important step in assessing program implementation and determining context for implementing evidence-based best practices.

Objective: The purpose of this process evaluation was to determine the extent to which the healthy corner store PSE initiative was implemented as intended and to identify facilitators and barriers to implementation in all seven participating stores during FY 2017.

Study Design, Setting, Participants, Intervention: The mixed methods design included review of SNAP-Ed program activity logs, discussion with SNAP-Ed staff and partners, and site visits.

Outcome Measures and Analysis: Fidelity was assessed by comparing quantified input, activity, and output data from activity logs with the program logic model and store-specific goals. Field notes were analyzed for themes related to program implementation successes and challenges.

Results: Three of the seven corner stores were new to the initiative in 2017, reflecting successful program efforts to build and sustain relationships with store owners. Program staff implemented (or continued to support) early stage goals (e.g. educational events and presentation and promotion practices to highlight healthful foods already available). However, changing store food offerings proved challenging. Staff cited cultural preferences, limited buying power, and limited store size as barriers to increasing fruit, vegetables, and low-fat dairy offerings.

Conclusions and Implications: This healthy corner store PSE initiative reflects nationwide evidence that sug- gests corner store programs can be slow-moving and require consistent effort and community buy-in. Process evaluation results indicated achievement of early program goals and highlighted challenges in creating sustainable changes in store food offerings. Results will immediately inform scheduled interviews with staff, community partners, store owners, and store customers and will inform interpretation of outcome results.

Funding: USDA.

\section{P181 Recipe Sampling and Recipe Card Distribution at Farmers' Markets Positively Correlated With Intent to Prepare Recipes Using Locally Grown Fruits and Vegetables}

Umaima Sidra Afsheen Syeda, MS, BS, University of Kentucky; Tammy Stephenson, PhD, tammy.stephenson@uky.edu, University of Kentucky, 202 Funkhouser Building, Lexington, KY 40504; Mikayla Cupp, BS; Jessica Houlihan, MPH, MSPAS, PA-C; Tharaka Galaniha, BS

Background (Background, Rationale, Prior Research, and/or Theory): The Plate It Up! Kentucky Proud (PIUKP) recipe development project, now in its 10th year, continues to promote purchase of fresh and seasonal locally grown fruits and vegetables. Recipes are developed by upper-level dietetics and nutrition students and tested by a trained panel and community volunteers. Recipe cards, educational materials, and samples are distributed by FCS Extension agents through community programming, farmers' markets, and other related community activities.

Objective: The purpose of this study was to determine the effects of recipe sampling and distribution of recipe cards on purchasing habits of consumers at diverse farmers' markets throughout Kentucky.

Study Design, Setting, Participants, Intervention: Surveys were conducted at farmers' markets across seven Kentucky counties $(n=223)$ in collaboration with local FCS Extension agents, who provided PIUKP recipe samples and professional cards.

Outcome Measures and Analysis: Twenty-two percent of the survey participants $(n=223)$ reported having tasted a PIUKP sample prior to this sampling experience. Participants reported consuming $2.39 \pm 1.24$ servings of fruits and $2.85 \pm 1.24$ servings of vegetables daily. The overall quality of the sampled recipe was rated as $8.63 \pm 1.71$ on a 10 -point Liker-scale with 10 being the "best" flavor. Recipe sampling influenced consumers' intent to purchase produce at the farmers' market that day $(\mathrm{r}=0.38 ; P<.0001)$ and in the future $(\mathrm{r}=0.413$; $P<.001)$. A positive correlation was also found between sampling $(\mathrm{r}=0.552 ; P<.0001)$ and recipe card distribution $(\mathrm{r}=0.25, P<.0002)$ and intent to prepare the PIUKP recipe at home.

Conclusions and Implications: Recipe sampling at farmers' markets provides consumers with an opportunity to learn about the health benefits of consuming fruits

Continued on page 594 